\title{
Validity and Reliability of Evaluation of Posture Using a Digital Inclination Meter
}

\author{
YoshikazU SuzUKi, MS, RPT ${ }^{1)}$, NAOTO KAMIDE, PhD, RPT ${ }^{2)}$, Kosuke MizUnO, MS, RPT ${ }^{1)}$, \\ KAYOKO TAKAHASHI-NARITA, ScD, OTR ${ }^{1)}$, YOSHIMI HIRAGA, MS, RPT ${ }^{1)}$, \\ MICHINARI FUKUDA, MD ${ }^{1,2)}$ \\ 1) Kitasato University East Hospital: 2-1-1 Asamizodai, Minami, Sagamihara, Kanagawa, 252-0380 \\ Japan.TEL: +81 42-748-2708, FAX: +81 42-748-2078,E-mail: yoshi.s@kitasato-u.ac.jp \\ ${ }^{2)}$ Kitasato University, School of Allied Health Sciences
}

\begin{abstract}
Purpose] Recently, a digital inclination meter was developed to evaluate severe defective posture. However, the validity and reliability of this instrument for slightly defective posture have not been tested. The purpose of this study was to test the intra-rater reliability, inter-rater validity and reliability of the instrument with healthy adults. [Methods] Ten subjects volunteered to participate in this study. Trunk and pelvis inclination angles were measured using the digital inclination meter. To test intra-rater reliability, a rater evaluated 5 standing postures 3 times for each of the 10 subjects using the digital inclination meter. To test inter-rater reliability, the postures of 5 subjects were measured 3 times by 3 raters. In order to test validity, each posture was also captured using a digital camera and image analysis software. [Results] All tests of intra-rater reliability and inter-rater reliability were at either the substantial or almost perfect level (intra-class correlation coefficients were from 0.61 to 1.00 ). Pearson's correlation coefficients for the results of digital inclination meter and image analysis were over 0.85 for all measurement items. [Conclusion] This evaluation method using the digital inclination meter was shown to be a reliable, valid and easy way to assess defective posture in a clinical setting as compared to image analysis using a digital camera and software.

Key words: Standing posture, Measurement instrument, Low back pain
\end{abstract}

(This article was submitted Nov. 10, 2010, and was accepted Dec. 11, 2010)

\section{INTRODUCTION}

Low back pain (LBP) is a common complaint across all ages. In fact, previous research has indicated that $70 \%$ of people experience of LBP of their lifetime ${ }^{1)}$. Severe LBP may make it difficult for people to perform activities of daily living, and prevent physical activity. As a result, LBP may cause disuse syndrome, such as muscle weakness. Various factors are thought to be the cause of LBP, and defective posture is one of them ${ }^{2}$. For example, if defective posture in sitting and standing is continued for a long period, myofascial LBP may be caused by an excessive stress on both dorsolumbar and abdominal muscles. Therefore, posture evaluation is important for physical therapists to prevent and manage LBP.

Observations by physical therapists, angle measurement of the trunk using a goniometer, and analysis of images captured by a digital camera have been used to clinically evaluate posture. However, methods of observation and measurement with goniometers have subjective evaluation issues, and depend on the rater's experience and technique $^{3)}$. On the other hand, analysis of images captured by a digital camera is objective and quantitative; however, it takes time to analyze and calculate the result from the digital image. Furthermore, because a digital camera can only shoot two dimensions of posture, this method is not able to measure defective posture involving a combination of flexion, extension, and lateral bending or rotation of trunk. Therefore, this method of using digital images is neither easy nor simple to perform in clinical practice.

Recently, a posture measurement device, an inexpensive digital inclination meter, was developed to evaluate defective posture in people with cerebral palsy. However, its validity and reliability has not been clarified for healthy adults or people with LBP who have mild posture defects as compared to people with cerebral palsy. The purpose of this study was to determine the validity and reliability of the measurement of posture of healthy adults using the digital inclination meter.

\section{SUBJECTS AND METHODS}

Ten volunteers ( 5 women: mean age $36.8 \pm 9.0$ years, mean height $155.4 \pm 9.3 \mathrm{~cm}$, mean weight $49.1 \pm 7.0 \mathrm{~kg} ; 5$ 
men: mean age $29.0 \pm 4.2$ years, mean height $175.1 \pm 6.4$ $\mathrm{cm}$, mean weight $71.6 \pm 11.8 \mathrm{~kg}$ ) participated in this study. Subjects with a history of orthopedic surgery or neurological disease were excluded. This study was approved by the Institutional Review Board of the School of Allied Health Sciences, Kitasato University (\#2010-012).

Inclination angles of the trunk and pelvis were measured using a digital inclination meter (Horizon; Yuki Trading, Inc. Tokyo, Japan) to evaluate of standing posture. The digital inclination meter has two arms that are placed on anatomical landmarks to measure the inclination angles of the trunk and pelvis, and it is able to measure to an accuracy of one degree. Measurement items are the forward and lateral inclination angles of the trunk, and the anterior and lateral inclination angles of the pelvis in standing. Raters measured inclination angles of the trunk and pelvis by putting the arms of the digital inclination meter on two anatomical landmarks as follows. Forward and lateral inclination angles of trunk were measured by using the superior and inferior margins of the sternum as landmarks. In measurement of lateral inclination angle of the pelvis, both sides of the anterior superior iliac spine were used as landmarks. In measurement of the anterior inclination angle of the pelvis, the right side of the anterior superior iliac spine and the right side of the posterior superior iliac spine were used as landmarks.

One rater (rater A) evaluated the standing postures of 10 subjects using the digital inclination meter in order to evaluate intra-rater reliability. Measurements were performed in 5 standing postures; (1) relaxed standing posture, (2) forward-bending standing posture, (3) backward-bending standing posture, (4) right-bending standing posture, (5) left-bending standing posture. The standing postures of (2) to (5) which was simulated for defective standing posture was defined by less than $20 \mathrm{~cm}$ of reach task to each direction. Each standing posture was measured three times by rater A.

Furthermore, to evaluate inter-rater reliability, 5 out of 10 participants were randomly selected to be evaluated by three raters (A, B, C). Rater A was a physical therapist (1 year of experience), rater B was an occupational therapist (2 years of experience), rater $\mathrm{C}$ was a speech therapist (4 years of experience). Prior to evaluation, all raters were thoroughly trained on how to use the digital inclination meter and how to check subject's anatomical landmarks. Relaxed standing posture of five subjects was measured three times by each rater. All raters measured each posture by the following procedures. First, rater indicated subject's anatomical landmarks with the arms of the device without looking at the device's display. Second, rater pushed a "HOLD" button to record the angle into the hardware of the device. Third, rater confirmed and read out the displayed angle in one degree unit.

For evaluation of validity, each standing posture described above was captured using a digital camera at the same time as measurement with digital inclination meter by rater A. Two digital cameras (DMC-FZ30; Panasonic inc. Osaka, Japan, DSC-T300; SONY inc. Tokyo, Japan) were used to capture standing posture in experimental setting; one camera was set in front of the subject to capture standing posture on the frontal plane, and the other camera was set on right side of the subject to capture it on the sagittal plane. Distance from subjects to cameras was set at 4 meters, and height from floor to the cameras was set at 1.1 meters. Five reflective markers were attached on both sides of acrominon, anterior superior iliac spine and right side of posterior superior iliac spine. Captured digital photographic images were analyzed using software, image J (National Institutes of Health; Maryland, USA), and inclination angles of trunk and pelvis were calculated. Validity and reliability of above method using digital camera and software had been shown in a previous study, and details of measurement and analysis method had been described elsewhere ${ }^{4,5)}$

Based on evaluation by rater $\mathrm{A}$, intra-rater reliability was analyzed using intra-class correlation coefficient (ICC (1, $3)$ ). Inter-rater reliability was analyzed using intra-class correlation coefficient $(\operatorname{ICC}(2,3))$. A reliability coefficient of between 0.41 and 0.60 is characterized as 'moderate' level, between 0.61 and 0.80 is characterized as 'substantial' level, 0.81 or above is characterized as 'almost perfect' level ${ }^{6}$. Validity was assessed by Pearson correlation coefficient between measurement values obtained from digital inclination meter and calculation values analyzed from the digital image. In addition, mean differences of trunk and pelvis inclinations angles between digital

Table 1. Mean inclination angle measured with digital inclination meter

\begin{tabular}{lcccc}
\hline \multirow{2}{*}{ Standing posture } & \multicolumn{2}{c}{ Trunk inclination } & \multicolumn{2}{c}{ Pelvis inclination } \\
\cline { 2 - 5 } & Lateral & Forward & Lateral & Anterior \\
\hline Relaxed standing & $-1.4 \pm 3.0$ & $-13.3 \pm 7.0$ & $-0.9 \pm 2.0$ & $6.3 \pm 6.3$ \\
Forward-bending & $0.7 \pm 4.1$ & $8.0 \pm 10.5$ & $0.1 \pm 1.9$ & $21.1 \pm 8.4$ \\
Backward-bending & $-3.6 \pm 3.6$ & $-32.4 \pm 8.4$ & $-0.3 \pm 2.2$ & $-2.6 \pm 6.7$ \\
Right-bending & $-22.7 \pm 9.1$ & $-8.5 \pm 7.0$ & $-4.4 \pm 4.1$ & $8.4 \pm 6.3$ \\
Left-bending & $18.2 \pm 10.3$ & $-11.3 \pm 7.2$ & $3.7 \pm 3.1$ & $4.1 \pm 5.4$ \\
\hline
\end{tabular}

$\mathrm{n}=10$, unit: degree

Note. Positive direction was indicated left-inclination of trunk, forward-inclination of trunk, lateral inclination of pelvis, anterior inclination of pelvis. 
Table 2. Intra-rater reliability in each posture and measurement item

\begin{tabular}{|c|c|c|c|c|c|c|c|c|}
\hline \multirow{3}{*}{ Standing posture } & \multicolumn{4}{|c|}{$\begin{array}{c}\mathrm{ICC}(1,1) \\
(95 \% \mathrm{CI})\end{array}$} & \multicolumn{4}{|c|}{$\begin{array}{c}\operatorname{ICC}(1,3) \\
(95 \% \mathrm{CI})\end{array}$} \\
\hline & \multicolumn{2}{|c|}{ Trunk inclination } & \multicolumn{2}{|c|}{ Pelvis inclination } & \multicolumn{2}{|c|}{ Trunk inclination } & \multicolumn{2}{|c|}{ Pelvis inclination } \\
\hline & Lateral & Forward & Lateral & Anterior & Lateral & Forward & Lateral & Anterior \\
\hline Relaxed standing & $\begin{array}{c}0.82 \\
(0.58-0.95)\end{array}$ & $\begin{array}{c}0.91 \\
(0.77-0.97)\end{array}$ & $\begin{array}{c}0.71 \\
(0.39-0.91)\end{array}$ & $\begin{array}{c}0.97 \\
(0.92-0.99)\end{array}$ & $\begin{array}{c}0.93 \\
(0.80-0.95)\end{array}$ & $\begin{array}{c}0.97 \\
(0.91-0.99)\end{array}$ & $\begin{array}{c}0.88 \\
(0.66-0.97)\end{array}$ & $\begin{array}{c}0.99 \\
(0.97-0.99)\end{array}$ \\
\hline Forward-bending & $\begin{array}{c}0.96 \\
(0.90-0.99)\end{array}$ & $\begin{array}{c}0.99 \\
(0.97-1.00)\end{array}$ & $\begin{array}{c}0.91 \\
(0.76-0.97)\end{array}$ & $\begin{array}{c}0.98 \\
(0.94-0.99)\end{array}$ & $\begin{array}{c}0.96 \\
(0.75-0.97)\end{array}$ & $\begin{array}{c}1.00 \\
(0.99-1.00)\end{array}$ & $\begin{array}{c}0.97 \\
(0.90-0.99)\end{array}$ & $\begin{array}{c}0.99 \\
(0.98-1.00)\end{array}$ \\
\hline Backward-bending & $\begin{array}{c}0.69 \\
(0.36-0.90)\end{array}$ & $\begin{array}{c}0.98 \\
(0.96-1.00)\end{array}$ & $\begin{array}{c}0.87 \\
(0.68-0.96)\end{array}$ & $\begin{array}{c}0.97 \\
(0.92-0.99)\end{array}$ & $\begin{array}{c}0.87 \\
(0.63-0.96)\end{array}$ & $\begin{array}{c}0.99 \\
(0.98-1.00)\end{array}$ & $\begin{array}{c}0.95 \\
(0.87-0.99)\end{array}$ & $\begin{array}{c}0.99 \\
(0.97-1.00)\end{array}$ \\
\hline Right-bending & $\begin{array}{c}0.93 \\
(0.83-0.98)\end{array}$ & $\begin{array}{c}0.95 \\
(0.87-0.99)\end{array}$ & $\begin{array}{c}0.78 \\
(0.51-0.93)\end{array}$ & $\begin{array}{c}0.97 \\
(0.96-1.00)\end{array}$ & $\begin{array}{c}0.98 \\
(0.93-0.99)\end{array}$ & $\begin{array}{c}0.98 \\
(0.95-1.00)\end{array}$ & $\begin{array}{c}0.91 \\
(0.76-0.98)\end{array}$ & $\begin{array}{c}1.00 \\
(0.99-1.00)\end{array}$ \\
\hline Left-bending & $\begin{array}{c}0.97 \\
(0.91-0.99)\end{array}$ & $\begin{array}{c}0.89 \\
(0.73-0.97)\end{array}$ & $\begin{array}{c}0.83 \\
(0.60-0.95)\end{array}$ & $\begin{array}{c}0.96 \\
(0.91-0.99)\end{array}$ & $\begin{array}{c}0.99 \\
(0.97-1.00)\end{array}$ & $\begin{array}{c}0.96 \\
(0.89-0.99)\end{array}$ & $\begin{array}{c}0.94 \\
(0.82-0.98)\end{array}$ & $\begin{array}{c}0.99 \\
(0.97-1.00)\end{array}$ \\
\hline
\end{tabular}

Note. All coefficients were $\mathrm{p}<0.01 .95 \% \mathrm{CI}$ was $95 \%$ confidence interval.

Table 3. Inter-rater reliability in each posture and measurement item

\begin{tabular}{|c|c|c|c|c|c|c|c|c|}
\hline \multirow{3}{*}{ Standing posture } & \multicolumn{4}{|c|}{$\begin{array}{c}\operatorname{ICC}(2,1) \\
(95 \% \mathrm{CI})\end{array}$} & \multicolumn{4}{|c|}{$\begin{array}{c}\operatorname{ICC}(2,3) \\
(95 \% \mathrm{CI})\end{array}$} \\
\hline & \multicolumn{2}{|c|}{ Trunk inclination } & \multicolumn{2}{|c|}{ Pelvis inclination } & \multicolumn{2}{|c|}{ Trunk inclination } & \multicolumn{2}{|c|}{ Pelvis inclination } \\
\hline & Lateral & Forward & Lateral & Anterior & Lateral & Forward & Lateral & Anterior \\
\hline \multirow{2}{*}{ Relaxed standing } & 0.62 & 0.93 & 0.34 & 0.73 & 0.83 & 0.98 & 0.61 & 0.89 \\
\hline & $(0.34-0.83)$ & $(0.79-0.98)$ & $(0.05-0.66)$ & $(0.27-0.91)$ & $(0.61-0.94)$ & $(0.92-0.99)$ & $(0.12-0.85)$ & $(0.50-0.97)$ \\
\hline
\end{tabular}

$\mathrm{n}=5$

Note. All coefficients were $\mathrm{p}<0.01 .95 \% \mathrm{CI}$ was $95 \%$ confidence interval.

Table 4. Pearson correlation coefficient between image analysis and digital inclination meter

\begin{tabular}{lcccc}
\hline & \multicolumn{2}{c}{ Trunk inclination } & \multicolumn{2}{c}{ Pelvis inclination } \\
\cline { 2 - 5 } & Lateral & Forward & Lateral & Anterior \\
\hline Correlation coefficient & 0.952 & 0.845 & 0.895 & 0.939 \\
$95 \%$ CI & $(0.91-0.97)$ & $(0.74-0.91)$ & $(0.82-0.94)$ & $(0.87-0.96)$ \\
\hline
\end{tabular}

$\mathrm{n}=50$

Note. All coefficients were $\mathrm{p}<0.01 .95 \% \mathrm{CI}$ was $95 \%$ confidence interval.

inclination meter and digital image were also calculated to assess measurement inconsistency. The statistical analyses were performed using SPSS for Windows, version 11.0J (SPSS Japan, Inc., Tokyo, Japan). The significance level was set at $5 \%$.

\section{RESULTS}

In each standing posture, trunk and pelvis inclination angle of all subjects was shown in Table1. Intra-rater reliability and inter-rater reliability by ICC were summarized in Table 2 and 3. All $\operatorname{ICC}(1,3)$ which assessed intra-rater reliability were in 'almost perfect' level. All ICC $(2,3)$ which assessed inter-rater reliability were in either 'substantial' or 'almost perfect' level. Pearson's correlation coefficients between digital inclination meter and digital image were summarized in Table 4 . There were significant high correlation coefficients $(\mathrm{r}=0.84-0.95, \mathrm{P}<0.01)$ between digital inclination meter and digital image in all 5 standing posture. Mean differences of trunk and pelvis inclinations angles between digital inclination meter and digital image were summarized in Table 5. Mean differences in trunk 
Table 5. Mean differences between digital image analysis and digital inclination meter

\begin{tabular}{lcccc}
\hline Standing posture & \multicolumn{2}{c}{ Trunk inclination } & \multicolumn{2}{c}{ Pelvis inclination } \\
\cline { 2 - 5 } & Lateral & Forward & Lateral & Anterior \\
\hline Relaxed standing & $2.92 \pm 1.22$ & $12.75 \pm 6.90$ & $1.27 \pm 1.13$ & $2.88 \pm 1.26$ \\
Forward-bending & $2.71 \pm 2.13$ & $15.24 \pm 7.77$ & $1.17 \pm 0.52$ & $3.58 \pm 2.37$ \\
Backward-bending & $3.70 \pm 4.47$ & $12.17 \pm 8.98$ & $1.45 \pm 0.99$ & $5.13 \pm 2.96$ \\
Right-bending & $7.59 \pm 3.85$ & $7.74 \pm 5.14$ & $1.94 \pm 1.39$ & $2.73 \pm 1.95$ \\
Left-bending & $5.83 \pm 2.97$ & $13.20 \pm 7.93$ & $2.24 \pm 121$ & $3.44 \pm 2.71$ \\
\hline
\end{tabular}

$\mathrm{n}=10$, unit: degree

forward inclination angle were larger than other inclination angle.

\section{DISCUSSION}

Intra-rater reliability of measurement using the digital inclination meter was shown to be at high level (ICC $(1,3)$ were over 0.87 ). Inter-rater reliability of measurement using the digital angle was also at 'almost perfect' level, excluding lateral inclination angles of pelvis which was at substantial level (ICC $(2,3)$ were over 0.61$)$. Therefore, it was shown that reliability of posture evaluation using the digital inclination meter is sufficient.

Validity was assessed by comparing the results of digital inclination meter with that of digital image analysis. As results, Pearson's correlation coefficient between digital inclination meter and digital image analysis were over 0.845 in all standing posture. Therefore, it was shown that posture evaluation using digital inclination meter has high validity. On the other hand, differences of the inclination angles of trunk and pelvis between digital inclination meter and digital image were less than 7 degree, except for trunk forward inclination angle. The cause of this large mean difference in trunk forward inclination was thought to be the difference of landmarks adapted to measure. In measurement of digital inclination meter, top and lower end of sternum was set as landmarks. In digital image analysis, acrominon and midway point between anterior superior iliac spine and right side of posterior superior iliac spine was set as landmark. That difference of landmarks could be the cause of large mean difference. However, since correlation coefficient between digital inclination meter and digital image analysis in trunk forward inclination was high $(\mathrm{r}=0.845)$, measurement accuracy was suggested to be sufficient. Therefore, it was concluded that the posture evaluation using the digital inclination meter is thought to be same quality to image analysis.

Comparing to posture evaluation of digital image analysis, it was suggested that using digital inclination meter has two main advantages. First, time which was necessary to measure by digital inclination meter was shorter than that by digital image analysis. If we evaluate a posture with digital image analysis, rater needs to spend about 10-20 minutes to output the result. In fact, process of digital image analysis is very complicated, i.e. rater sets digital cameras, capture a posture by cameras, import images to a personal computer and analyze digital images. On the contrary, for evaluation of digital inclination meter we found that rater could measure in about one minute, since operation of the device was very simple. Second, the digital inclination meter does not need larger space for measurement setting as compared to digital image analysis. Space of more than 3 square meters is necessary for digital image analysis to set up digital cameras; however, digital inclination meter is portable device which could be used at narrow space. Therefore, from the perspective of time and space for measurement procedure, digital inclination meter is considered to be more applicable in clinical setting as compared to other general methods for posture evaluation such as digital image analysis.

This work was a pilot study aimed to confirm validity and reliability of the new device. Therefore, present study had several limitations. First, it was unclear whether angle evaluated by digital inclination meter agree with spinal alignment assessed by X-ray image, since image analysis was used for evaluation of posture, not spinal alignment. Second, inter-rater reliability was lower than intra-rater reliability in ICC of single measure $\operatorname{ICC}(2,1)$ and ICC $(1,1))$. This result suggested that there were gaps between raters in the angles measured by digital inclination meter. Therefore, more regulation on measurement protocol may be necessity to improve accuracy of the measurement value. We recommend the procedure used in this study for clinical use: measurement is taken multiple times ( 2 times or more) and the average of those values is used. Third, people who had deformity of chest or spine and instability of standing balance were not included in the present study. Therefore, application of posture evaluation using digital inclination meter on those people must be considered carefully.

In conclusion, the posture evaluation using a digital inclination meter showed high intra-rater and inter-rater reliability and high validity. And, since posture evaluation with digital inclination meter is able to evaluate simply and quickly as compared to digital image analysis, it is clinically useful.

\section{REFERENCES}

1) Frymoyer JW, Pope MH, Clements JH, et al.: Risk factors in low-back pain. An epidemiological survey. J Bone Joint Surg Am. 1983, 65: 213-218.

2) Roffey DM, Wai EK, Bishop P, et al. : Causal assessment of awkward 
occupational postures and low back pain: results of a systematic review. Spine J. 2010, 10: 89-99.

3) Fedorak C, Ashworth N, Marshall J, et al.: Reliability of the visual assessment of cervical and lumbar lordosis: how good are we? Spine. 2003, 28: $1857-1859$.

4) Ferreira EA, Duarte M, Maldonado EP et al.: Postural assessment software
(PAS/SAPO): Validation and reliability. Clinics. 2010, 65: 675-681.

5) National Institutes of Health. U.S. Department of Health \& Human Services http://rsb.info.nih.gov/ij/ (Accessed Oct.30, 2010)

6) Landis JR, Koch GG: The measurement of observer agreement for categorical data.1977, 33: 159-174. 